\title{
LINGUAGEM E MEMÓRIA NA DOENÇA \\ DE ALZHEIMER EM FASE MODERADA
}

\section{Language and memory in the Alzheimer's disease on the moderate phase}

\author{
Patricia Gomes de Azevedo (1), Mirela Espessoto Landim (2), \\ Gisele Priscila Fávero ${ }^{(3)}$, Ana Lúcia de Magalhães Leal Chiappetta ${ }^{(4)}$
}

\begin{abstract}
RESUMO
Objetivo: analisar as alterações cognitivas (memória e linguagem) no paciente com doença de Alzheimer na fase moderada e verificar se as variáveis sexo, idade e escolaridade interferem nessas habilidades. Métodos: foi realizado um estudo observacional e prospectivo com 27 sujeitos com doença de Alzheimer na fase moderada, frequentadores do Centro de Reabilitação Regional de Araraquara (CRRA), com idade variando de 60 a 86 anos. O teste utilizado para a avaliação foi - Consortium to Establish a Registry for Alzheimer's Disease (CERAD) que contém várias provas, sendo selecionadas as seguintes: Teste de nomeação de Boston, Memória da lista de palavras, Fluência verbal, Evocação da lista de palavras e Reconhecimento da lista de palavras. Resultados: as respostas obtidas nesta pesquisa permitiram observar que não houve diferença significante nas variáveis sexo e idade; já na variável escolaridade, obteve-se diferença significante no subteste de nomeação de Boston. Conclusão: a amostra dos sujeitos desta pesquisa não obteve diferença significante nas variáveis sexo e idade. Já na variável escolaridade, os sujeitos com maior grau de instrução tiveram melhor desempenho na prova de linguagem relacionada ao subteste de nomeação de Boston.
\end{abstract}

DESCRITORES: Doença de Alzheimer; Memória; Linguagem

\section{INTRODUÇÃO}

Com o aumento do envelhecimento da população mundial, as demências vêm crescendo, tendo como causa mais comum a doença de Alzheimer, que é uma doença progressiva que degenera as funções neurais - degeneração neurofibrilar, particularmente no neocortex, hipocampo

(1) Fonoaudióloga; Especializanda em Linguagem do CEFAC - Pós-Graduação em Saúde e Educação.

(2) Fonoaudióloga do Centro Auditivo Sense, Ribeirão Preto, SP; Especializanda em Linguagem do CEFAC - Pós-Graduação em Saúde e Educação.

(3) Fonoaudióloga da União dos Deficientes Físicos, UDF, Araraquara, SP; Especializanda em Linguagem do CEFAC - Pós-Graduação em Saúde e Educação.

(4) Fonoaudióloga do Setor de Investigação em Doenças Neuromusculares da Universidade Federal de São Paulo, UNIFESP, São Paulo, SP; Doutora em Ciências pela Universidade Federal de São Paulo.

Conflito de interesses: inexistente (onde ocorre o início do distúrbio da memória) e amígdala, sendo uma de suas principais causas a perda da capacidade funcional em idosos, ou seja, a demência ${ }^{1-4}$.

A doença de Alzheimer (DA) caracteriza-se por uma perda gradativa de memória e de outras funções cognitivas, resultando em déficits nas atividades de vida diária, sociais e ocupacionais do indivíduo. As terapias disponíveis para a D.A. não diminuem o curso da doença, porém os tratamentos podem intervir atrasando a manifestação clínica da mesma. A DA representa $50-70 \%$ das doenças demenciais. Sua prevalência é 3-5\% nas pessoas com mais de 65 anos e sua incidência é de 1-2\% ao ano na sua população geral ${ }^{5-10}$.

Para se diagnosticar a doença de Alzheimer é necessário que, além do comprometimento da memória, ocorra pelo menos mais um déficit da função cognitiva, como linguagem, atenção seletiva e dividida, e funções executivas, e essas informações só podem ser obtidas por meio de avaliações 
diretas com o paciente ou com entrevistas realizadas com o cuidador ${ }^{11}$.

A neuropatologia da DA carateriza-se por dois mecanismos críticos que determinam a morte neuronal: 1) formação de placas amilóides pela gamasecretase e beta-secretase; 2) hiperfosforilação da proteína tau, que leva à formação de emaranhados neurofibrilares dentro dos neurônios. Estes mecanismos determinam o processo de atrofia cerebral, inicialmente em áreas mesiais do lobo temporal, como hipocampo e córtex entorrinal - áreas associadas ao processamento de memória recente - e atrofia do núcleo de Meynert, bem como dos núcleos septais, no prosencéfalo basal. Estes núcleos são responsáveis pela produção de acetilcolina, um neurotransmissor mediador da atividade cognitiva. Progressivamente, o processo neurodegenerativo acomete todo o córtex cerebral, determinando o declínio das demais funções cognitivas, além de distúrbios de comportamento. As mudanças neuropatológicas ocorrem antecipadamente ao diagnóstico clínico da doença de Alzheimer; mesmo assim, a capacidade cerebral pode ser compensada até o momento em que a função cognitiva comece a apresentar falhas evidentes ${ }^{12-20}$.

Existem 3 fases na Doença de Alzheimer com aproximadamente 2 a 3 anos cada. Na fase primária, leve, ou inicial, ocorre um déficit na memória recente e remota, com alterações na personalidade, alternando estágios de irritabilidade, hostilidade, apatia e frustração. Na comunicação, o sujeito apresenta desordens no conteúdo da linguagem, associadas a déficit no raciocínio linguístico e disfonia; possui dificuldades em buscar a palavra correta ou lembrar nomes de objetos ou pessoas. As habilidades visuoespaciais também se apresentam alteradas, havendo construções incorretas e desordenação topográfica. O paciente não é capaz de solucionar problemas, apresenta-se confuso e tem dificuldade em tomar decisões. O sistema motor geralmente está normal, com alguns sinais extrapiramidais. Ainda nesse estágio, o paciente possui consciência e percepção de suas dificuldades e usa, frequentemente, recursos ou estratégias para compensá-las ${ }^{21-25}$.

Na fase secundária, moderada, ou intermediária, acentua-se o sensível déficit de memória e aprendizagem. Também ocorrem mudanças de personalidade, indiferença, hostilidade, julgamento social pobre, baixa afetividade. A comunicação apresenta um conteúdo desorganizado e alguns déficits estruturais que prejudicam a coerência; possui uma desorientação espacial, construção pobre, dificuldades perceptivas e, nessa fase, o período de fala é mais fluente, porém menos coerente, apresentando agitação e, no sistema motor de fala, apresenta tremores ou cacoetes ${ }^{21-25}$.

$\mathrm{Na}$ fase terciária, grave, ou final, temos as funções intelectuais globalmente deterioradas, um estado de dependência total. Sua personalidade mostra-se totalmente desorganizada, a comunicação está deteriorada com ecolalias, perseveração e mutismo. É totalmente dependente de outros para solucionar problemas e realizar atividades diárias, higiene pessoal, alimentação. Apresenta rigidez na região dos quadris e postura em flexão, conhecida como a síndrome da imobilização ${ }^{21-25}$.

Uma das dificuldades para se avaliar a cognição é a falta de padronização de testes. O CERAD (Consortium to Establish a Registry for Alzheimer's Disease), que foi desenvolvido pelo departamento de Neurologia da Universidade de Lisboa, o qual possui uma versão brasileira desenvolvida pela disciplina de Neurologia e Geriatria UNIFESP-EPM (Universidade de SP - Escola Paulista de Medicina) em São Paulo, tem a possibilidade de comparar resultados entre grupos e estudos diferentes, como pessoas normais envelhecidas e com provável doença de Alzheimer ${ }^{1,26}$.

Desta forma, o objetivo do presente estudo é analisar as alterações cognitivas (memória e linguagem) no paciente com doença de Alzheimer na fase moderada e verificar se as variáveis sexo, idade e escolaridade interferem nessas habilidades.

\section{MÉTODOS}

Foi realizado um estudo prospectivo e transversal durante o $1^{\circ}$ semestre de 2007 no CRRA (Centro Regional de Reabilitação de Araraquara - setor do idoso), com uma amostra constituída por 27 sujeitos (9 homens e 18 mulheres) na faixa etária de 60 a 86 anos com DA provável. Por meio do teste neuropsicológico Mini-Exame do Estado Mental (MEEM) realizado pelos médicos e psicólogos do CRRA para comprovar a provável DA e realizar os atendimentos trimestrais, foram excluídos da pesquisa os sujeitos com grau leve e grave da doença, e, foram incluídos os indivíduos com grau moderado. Por isso, não foi utilizado o CDR (Clinical Dementia Rating) para realmente comprovar em que fase da doença de Alzheimer os sujeitos se encontravam. $O$ teste utilizado para a realização desta pesquisa foi - Consortium to Establish a Registry for Alzheimer's Disease (CERAD), que contém várias provas, das quais foram selecionadas as seguintes: Teste de nomeação de Boston, Memória da lista de palavras, Fluência verbal, Evocação da lista de palavras e Reconhecimento da lista de palavras.

Teste de nomeação de Boston: foram apresentadas 15 figuras do Teste de Nomeação de Boston 
(árvore, cama, apito, flor, casa, canoa, escova de dente, vulcão, máscara, camelo, gaita, pegador de gelo, rede, funil, dominó). São consideradas apenas as respostas corretas obtidas sem pistas. $O$ escore máximo para este teste é 15 pontos. Memória da lista de palavras: 10 palavras não relacionadas foram apresentadas uma a uma para serem lidas em voz alta pelo sujeito (ou pesquisador, caso a leitura esteja prejudicada), sendo uma palavra a cada 2 segundos. Durante 90 segundos o sujeito terá que recordar as 10 palavras que foram lidas. As mesmas palavras serão apresentadas em outra ordem, mais duas vezes. A pontuação é obtida pela soma das palavras evocadas nas 3 tentativas, com um escore máximo de 30 pontos.

Fluência verbal: é dado o comando: "Fale todos os animais que conseguir lembrar. Vale qualquer tipo de bicho". Depois de dadas as ordens, é contado um minuto e o escore é quantificado de acordo com o número de animais lembrados nesse período. Animais cuja denominação de gênero é similar (p. ex. gato e gata), um deles não é pontuado, mas quando a denominação é diferente (p. ex. cavalo e égua), ambos são pontuados. A denominação genérica de subcategorias de animais é pontuada quando não seguida por exemplos da classe (p.ex. peixe ou pássaro); quando não é considerada a denominação de classe, são contados os exemplos. Dessa forma, os exemplos "gato, cavalo, peixe, vaca" receberiam 4 pontos, e a sequência "gato, gata, peixe, tubarão, baleia" receberia 3 pontos.

Evocação da lista de palavras: a evocação da lista de palavras foi apresentada previamente, por um período máximo de 90 segundos, com um escore máximo de 10 pontos. Nesta etapa, o sujeito terá que lembrar as 10 palavras que leu na prova de Memória da lista de palavras.

Reconhecimento da lista de palavras: após a evocação espontânea, as 10 palavras são apresentadas misturadas a 10 novas palavras. O número máximo de respostas corretas é 20 , mas o escore máximo é 10, pois o escore é calculado pela subtração de 10 do número de respostas corretas (corrigindo o efeito de respostas ao acaso).

Esta pesquisa foi avaliada pelo Comitê de Ética em Pesquisa do CEFAC - Saúde e Educação e aprovada sob o número 018/07.

Os resultados do estudo foram analisados pelo programa GraphPad Instat ${ }^{\circledR}$, version 3.05
(GraphPad, Inc., San Diego, CA, USA). A distribuição dos dados foi verificada pelo teste de normalidade de Kolmogorov-Smirnov. Os dados foram expressos em médias ( \pm desvios padrão). Para a comparação da análise intergrupos, utilizou-se o teste t de Student e o nível de significância considerado foi de $p \leq 0,05$ para a aplicação de todos os testes estatísticos.

\section{RESULTADOS}

Segundo os dados obtidos através dos subtestes realizados com os 27 sujeitos, a Tabela 1 demonstra a caracterização da amostra pesquisada, cuja idade (anos) apresentou valores em média de 76,9 e desvio padrão de 6,7 com variação mínima de 60 e máxima de 86 . Quanto à variável sexo, foram avaliadas 18 mulheres e 9 homens com variação de 0 a 1. Com relação à variável escolaridade, 8 sujeitos são alfabetizados e 12 analfabetos, variando de 0 a 1. Quanto à variável tempo de escolaridade (anos), os valores em média foram 4,4 e desvio padrão de 4,1 com variação de 0 a 12 . De acordo com as provas aplicadas, na de fluência verbal observaram-se valores em média de 6,8 com desvio padrão de 3,4 e variação de 0 a 12; no subteste de nomeação de Boston, os valores em média foram de 7,8 e desvio padrão de 2,2 com variação de 2 a 12; na prova de memória da lista de palavras, a média foi de $8,5 \mathrm{e}$ desvio padrão de 4,6, variando de 0 a 16; no subteste de evocação da lista de palavras, os valores em média foram de 2,2 e desvio padrão de 1,5 com variação de 0 a 5 e o subteste de reconhecimento verbal apresentou valores em média de 4,5 e desvio padrão de 3,0 com variação de 0 a 10 .

A Tabela 2 mostra os valores da média e desvio padrão referente a cada subteste aplicado na comparação por gênero, no qual não houve diferença significante entre homens e mulheres.

A Tabela 3 mostra os valores da média e desvio padrão referente a cada subteste aplicado na comparação por faixa etária, no qual também não houve diferença significante entre os com 60 a 77 anos, e os com 78 a 86 anos.

A Tabela 4 mostra os valores da média e desvio padrão referente a cada subteste aplicado na comparação por faixa de escolaridade, no qual a faixa de escolaridade de 7 a 12 anos obteve diferença significante no subteste de Boston $(p=0,0038)$. 
Tabela 1 - Caracterização da Amostra

\begin{tabular}{lccc}
\hline & Variáveis & $\begin{array}{c}\text { IC 95\% (valores } \\
\text { inferior- superior) }\end{array}$ & $\begin{array}{c}\text { Variação (valores } \\
\text { mínimo - máximo) }\end{array}$ \\
\hline Sujeitos, $n$ & 27 & & $0-1$ \\
Sexo, feminino: masculino & $18: 9^{*}$ & & $0-1$ \\
Alfabeto: analfabeto & $8: 12^{*}$ & $74,3-79,5$ & $60,0-86,0$ \\
Idade, anos & $76,9 \pm 6,7$ & $2,8-6,0$ & $0-12$ \\
Tempo Escolaridade, anos & $4,4 \pm 4,1$ & $5,5-8,1$ & $1-15$ \\
Fluência Verbal & $6,8 \pm 3,4$ & $6,9-8,6$ & $2-12$ \\
Nomeação de Boston & $7,8 \pm 2,2$ & $6,7-10,3$ & $0-16$ \\
Memória da Lista de Palavra & $8,5 \pm 4,6$ & $1,6-2,8$ & $0-5$ \\
Evocação da Lista & $2,2 \pm 1,5$ & $0,6-5,7$ & $0-10$ \\
Reconhecimento Verbal & $4,5 \pm 3,0$ & & \\
\hline
\end{tabular}

*Valores em média \pm desvio padrão, exceto onde indicado. IC: intervalo de confiança de $95 \%$.

Tabela 2 - Medidas da média e desvio-padrão referente a cada subteste na comparação por sexo

\begin{tabular}{lccc}
\hline Testes & Feminino & Masculino & p \\
\hline Teste de nomeação de Boston & $7,4 \pm 2,1$ & $8,4 \pm 2,1$ & 0,26 \\
Memória da lista de palavras & $7,9 \pm 4,8$ & $9,8 \pm 4,2$ & 0,32 \\
Fluência verbal & $6,5 \pm 3,1$ & $7,3 \pm 3,9$ & 0,55 \\
Evocação da lista de palavras & $2,3 \pm 1,7$ & $2,0 \pm 1,3$ & 0,60 \\
Reconhecimento da lista de palavras & $4,7 \pm 3,0$ & $4,0 \pm 3,0$ & 0,56 \\
\hline
\end{tabular}

Teste t de Student não-pareado: ${ }^{*} \mathrm{p} \leq 0,05$

Tabela 3 - Medidas da média e desvio-padrão referente a cada subteste na comparação por idade

\begin{tabular}{lccc}
\hline Testes & $\mathbf{6 0 - 7 7}$ anos & $\mathbf{7 8 - 8 6}$ anos & $\mathbf{p}$ \\
\hline Teste de nomeação de Boston & $7,5 \pm 2,4$ & $8,1 \pm 1,9$ & 0,5 \\
Memória da lista de palavras & $10,0 \pm 4,2$ & $6,9 \pm 4,6$ & 0,08 \\
Fluência verbal & $6,9 \pm 3,5$ & $6,7 \pm 3,4$ & 0,9 \\
Evocação da lista de palavras & $2,4 \pm 1,4$ & $2,1 \pm 1,7$ & 0,64 \\
Reconhecimento da lista de palavras & $5,4 \pm 2,9$ & $3,5 \pm 2,8$ & 0,11 \\
\hline
\end{tabular}

Teste t de Student não-pareado: ${ }^{*} \mathrm{p} \leq 0,05$

Tabela 4 - Medidas da média e desvio-padrão referente a cada subteste na comparação por escolaridade

\begin{tabular}{lccc}
\hline Testes & $\mathbf{0 - 6}$ anos/esc. & $\mathbf{7 - 1 2}$ anos/esc. & p \\
\hline Teste de nomeação de Boston & $7,1 \pm 1,8$ & $9,5 \pm 1,9$ & $0,0038^{*}$ \\
Memória da lista de palavras & $7,9 \pm 4,5$ & $10,0 \pm 4,8$ & 0,28 \\
Fluência verbal & $6,2 \pm 3,2$ & $8,3 \pm 3,4$ & 0,14 \\
Evocação da lista de palavras & $2,3 \pm 1,6$ & $2,0 \pm 1,5$ & 0,63 \\
Reconhecimento da lista de palavras & $4,2 \pm 2,9$ & $5,1 \pm 3,3$ & 0,48 \\
\hline
\end{tabular}

Teste t de Student não-pareado: ${ }^{*} \mathrm{p} \leq 0,05$ 


\section{DISCUSSÃO}

O envelhecimento populacional tem sido observado mundialmente, e isso não tem sido diferente no Brasil, pois seu perfil demográfico tem mudado, principalmente nas últimas décadas ${ }^{27,28}$. A população de idosos do Brasil vem aumentando, como provam os dados do censo de 2000 (IBGE- Instituto Brasileiro Geográfico e Estatístico), mostrando que 14,5 milhões de indivíduos encontram-se na faixa etária de 60 anos ou mais ${ }^{29,30}$; e que os hábitos da civilização vêm mudando, favorecendo o aumento das doenças cerebrais ${ }^{31}$, pois o estresse, cansaço mental, a falta de exercício físico, mental, e uma alimentação não tão saudável, favorecem as demências, em particular a doença de Alzheimer. A doença de Alzheimer é a forma mais comum de demência na terceira idade, afetando mais de 5\% da população com 65 anos de idade ${ }^{32}$.

As respostas obtidas nesta pesquisa permitiram observar que não houve diferença significante nas variáveis sexo e idade. Já na variável escolaridade, obteve-se diferença significante no subteste de nomeação de Boston.

Quanto à variável sexo, os sujeitos com doença de Alzheimer não obtiveram diferença significante nos subtestes aplicados, já que a prevalência ocorre nas mulheres. A associação entre o baixo nível de escolaridade e um aumento do risco de demência, ou doença de Alzheimer, foi mais evidente em mulheres do que em homens, nas faixas etárias dos 75 aos 84 anos do que na faixa etária com mais de 85 anos $^{33}$. Mas, nesta pesquisa, mesmo havendo um número maior de mulheres e com idade superior à dos homens, os dados coletados não comprovam pior desempenho das mesmas, sendo necessária uma pesquisa mais detalhada para outras análises.

Nesta pesquisa, a variável idade (60 a 86 anos) não interferiu no desempenho dos sujeitos, mesmo o envelhecimento sendo um fator de risco e a prevalência com relação à faixa etária seja aos 65 anos, sendo que aos 80 anos aumenta ainda mais o risco da doença de Alzheimer. Devem ser considerados outros fatores, além da idade, que possam estar interferindo no desempenho dos mesmos, como o comprometimento da memória, a linguagem, a atenção, a capacidade para resolver problemas ${ }^{34}$, o tratamento farmacológico, que, segundo pesquisas, mostra melhoras ou estabilização por períodos variáveis e uma reabilitação cognitiva ${ }^{35}$. É necessária assim, uma análise qualitativa considerando esses fatores.
$\mathrm{Na}$ variável escolaridade, os sujeitos com mais anos de estudo (7-12 anos) tiveram desempenho significante em um único subteste (teste de nomeação de Boston). Alguns pesquisadores encontraram um aumento de risco para doença de Alzheimer em indivíduos com um grau de escolaridade igual ou menor do que 8 anos, enquanto outros pesquisadores não conseguiram encontrar nenhuma evidência em relação à escolaridade ${ }^{36}$. É importante ressaltar que, nesta pesquisa, a maioria dos sujeitos $(n=20)$ eram analfabetos. Pesquisas relatam que a ocorrência de demência é mais frequente nos sujeitos analfabetos, quando comparados com os alfabetizados ${ }^{28,37}$. Embora o efeito da educação sobre o risco de demência e doença de Alzheimer ainda seja discutido ${ }^{36}$, já que as aquisições de conhecimentos criam novas conexões entre os neurônios (sinapses) e aumentam a reserva intelectual, ocasionando fatores que retardam o aparecimento das manifestações na demência.

Houve uma limitação em relação aos dados qualitativos, pois os sujeitos eram encaminhados para a pesquisa por meio da assistente social e dos médicos, ou após a realização das consultas trimestrais, com data e hora marcada. Com isso, não houve a realização do CDR para verificar se os sujeitos estavam realmente na fase moderada da doença de Alzheimer.

\section{CONCLUSÃO}

De acordo com os resultados obtidos, observouse, que na comparação por sexo e idade, os dados não foram significantes.

$\mathrm{Na}$ comparação por escolaridade, os sujeitos com mais anos de escolaridade obtiveram melhor desempenho em um dos subtestes que avaliavam a linguagem (teste de nomeação de Boston).

Como a maioria dos dados desta pesquisa não foi significante, talvez, com uma amostra maior de sujeitos, juntamente com os dados qualitativos, e complementando com os quantitativos, os resultados poderiam ser mais relevantes.

Vale ressaltar que, mesmo havendo várias pesquisas envolvendo pacientes com DA e seus cuidadores, ainda se faz necessário haver mais conhecimentos e esclarecimentos sobre a doença para os cuidadores e os profissionais envolvidos no que diz respeito às áreas comprometidas, à equipe multidisciplinar (geriatra, neurologista, fonoaudiólogo, entre outros); e, principalmente, um diagnóstico e reabilitação precoces. 


\section{ABSTRACT}

Purpose: to analyze the cognitive alterations (memory and language) in the patient with illness of Alzheimer in the moderate phase and to verify if the variables: gender, age and education interfere with these abilities. Methods: an observational study and prospective was carried through with 27 citizens with Alzheimer's disease in the moderate phase, patients of the Center of Regional Rehabilitation of Araraquara (CRRA), with age varying between 60 to 86 years. The test used for the evaluation was the Consortium to Establish a Registry for Alzheimer's Disease (CERAD) that contain some tests of which we select the following ones: Boston nomination test, Memory of list of words, Verbal Fluency, Calling up list of words and Recognition of list of words. Results: the answers obtained in this research allowed to observe that there was no significant difference in the variable gender and age; however, in the education variable, we got a significant difference in the Boston nomination subtest. Conclusion: the sample of the citizens of this research did not get significant difference in the variable gender and age. However, in the education variable, the subjects with higher educational degree had better performance in the language test related to the Boston nomination subtest.

KEYWORDS: Alzheimer Disease; Memory; Language

\section{REFERÊNCIAS}

1. Bertolucci PHF, Okamoto $\mathrm{IH}$, Toniolo Neto J, Ramos LR, Brucki SMD. Desempenho da população brasileira na bateria neuropsicológica do Consortium to Establish a Registry for Alzheimer's Disease (CERAD). Psiquiatr Clin. 1998; 25(2):80-3. 2. Abreu ID, Forlenza OV, Barros HL. Demência de Alzheimer: correlação entre memória e autonomia. Psiquiatr Clin. 2005; 32(3):131-6.

3. Carvalho ETF, Netto MP. Geriatria: fundamentos, clínica e terapêutica. In: Levy JA, Mendonça LI. Envelhecimento cerebral: demências. São Paulo: Atheneu; 2000. p. 51-62.

4. Lautenschlager NT. Is it possible to prevent dementia?. Rev Bras Psiquiatr. 2002; 24(1):22-7.

5. Alvarez AM, Ávila R, Carvalho IAM. Reabilitação neuropsicológica na doença de Alzheimer. Psiquiatr Clin. 2001; 28(6):286-7.

6. Behrens MIP, Vergara FE. Cien años de la enfermedad de Alzheimer. La inmunoterapia una esperanza?. Rev Méd Chile. 2007; 135(1):103-10.

7. Hueb TO. Doença de Alzheimer. Rev Bras Med. 2008; 65(4):90-5.

8. Almeida OP. Can we prevent Alzheimer's disease? Rev Bras Psiquiatr. 2005; 27(4):264-5.

9. Pérez MP. Las intervenciones dirigidas a los cuidadores de adultos mayores con enfermedad de Alzheimer. Rev Habanera Cienc Méd. 2008; 7(3):1-10.

10. Malagón C, Rodríguez J, Hernández J, Pardo $R$. Análisis del desempeño del lenguaje en sujetos con demencia tipo Alzheimer (DTA). Rev Fac Méd. 2005; 53(1):3-9.
11. Nitrini $R$, Caramelli $P$, Bottino $C M C$, Damasceno PB, Brucki SMD, Anghinah R. Diagnóstico de doença de Alzheimer no Brasil: avaliação cognitiva e funcional. Recomendações do Departamento Científico de Neurologia Cognitiva e do Envelhecimento da Academia Brasileira de Neurologia. Arq Neuropsiquiatr. 2005; 63(3A):720-7.

12. Coelho FGM, Santos-Galduroz RF, Gobbi $\mathrm{S}$, Stella F. Atividade física sistematizada e desempenho cognitivo em idosos com demência de Alzheimer: uma revisão sistemática. Rev Bras Psiquiatr. 2009; 31(2):163-70.

13. Nitrini $R$, Caramelli $P$, Mansur $L$, organizadores. Neuropsicologia: das bases anatômicas à reabilitação. São Paulo: Clínica Neurológica, Hospital das Clínicas, FMUSP; 2003

14. Grossman H, Bergmann C, Parker S. Dementia: a brief review. Mt Sinai J Med. 2006; 73(7): 985-92.

15. GauthierS, VellasB, FarlowM, BurnD. Aggressive course of disease in dementia. Alzheimer's \& Dementia. 2006; 2(3):210-7.

16. Donoso A, Behrens MI, Venegas P. Deterioro cognitivo leve: seguimiento de 10 casos. Rev Chil Neuro-Psiquiatr. 2003; 41(2):117-22.

17. Souza JN, Chaves EC, Caramelli P. Coping in aged people with Alzheimer's disease. Rev Latino-Am Enferm. 2007; 15(1):93-9.

18. Friedland RP, Fritsch T, Smyth KA, Koss E, Lerner AJ, Chen $\mathrm{CH}$, et al. Patients with Alzheimer's disease have reduced activities in midlife compared with healthy control-group members. Proc Natl Acad Sci. 2001; 98(6):3440-5. 
19. Twamley EW, Ropacki SA, Bondi MW. Neuropsychological and neuroimaging changes in preclinical Alzheimer's disease. J Int Neuropsychol Soc. 2006; 12(5):707-35.

20. Papassotiropoulos A, Fountoulakis $M$, Dunckley T, Stephan DA, Reiman EM. Genetics, transcriptomics and proteomics of Alzheimer's disease. J Clin Psychiatr. 2006; 67(4):652-70.

21. Costa EG, Silva MCC, Costa MLG, Barros ALS, Soares RF. Análise da deglutição em sujeitos portadores de doença de Alzheimer. Rev Bras Otorrinolaringol. 2008; 74(1). Suplemento.

22. Russo ICP. Intervenção fonoaudiológica na terceira idade. Rio de Janeiro: Revinter; 2004. p.136.

23. Rockland A, Borba J. Primeiros passos na fonoaudiologia: conhecer para intervir nas patologias, distúrbios e exames fonoaudiológicos. Recife: FASA; 2005. p. 200.

24. Gallucci Neto J, Tamelini MG, Forlenza OV. Diagnóstico diferencial das demências. Psiquiatr Clin. 2005; 32(3):119-30.

25. Lopera VEA. Dilemas éticos en etapas leves de la enfermedad de Alzheimer decirle o no la verdad al paciente. Rev Latinoam Bioét. 2008; 8(14): 58-65.

26. Bertolucci PHF, Okamoto $\mathrm{IH}$, Brucki SMD, Siviero MO, Toniolo Neto J, Ramos LR. Applicability of the CERAD neuropsychological battery to Brazilian elderly. Arq Neuropsiquiatr. 2001; 59(3A): 532-6.

27. Luzardo AR, Gorini MIPC, Silva APSS. Características de idosos com doença de Alzheimer e seus cuidadores: uma série de casos em um Serviço de Neurogeriatria. Texto Contexto Enferm. 2006; 15(4):587-94.

28. Vermelho LL, Monteiro MFG. Transição demográfica e epidemiológica. In: Medronho RA, Carvalho DM, Bloch KV, Luiz RR, Werneck GL. Epidemiologia. São Paulo: Atheneu; 2004. p. 91-103.
29. Garrido R, Menezes PR. Impacto em cuidadores de idosos com demência atendidos em um serviço psicogeriátrico. Rev Saúde Públ. 2004; 38(6):835-41.

30. Herrera Júnior E, Caramelli $P$, Nitrini R. Estudo epidemiológico populacional de demência na cidade de Catanduva, Estado de São Paulo, Brasil. Psiquiatr Clín. 1998; 25(2):70-3.

31. González JH, Galdames DC, Oporto SS. Perfiles diagnósticos y epidemiológicos en una Unidad de Memoria. Rev Méd Chile. 2005; 133(7):789-94.

32. Letenneur L, Launer J, Andersen K, Dewey ME, Ott A, Copeland JRM, et al. Education and the risk for Alzheimer's disease: sex makes a difference. Eurodem pooled analyses. Am J Epidemiol. 2000; 151(11):1064-71.

33. Qiu C, Bäckman L, Winblad B, Agüero-Torres H, Fratiglioni $L$. The influence of education on clinically diagnosed dementia incidence and mortality data from the Kungsholmen Project. Arch Neurol. 2001; 58(12):2034-9.

34. Caramelli P, Barbosa MT. Como diagnosticar as quatro causas mais freqüentes de demência?. Rev Bras Psiquiatr. 2002 abr; 24(Supl1):7-10.

35. Engelhardt E, Brucki SMT, Cavalcanti JLS, Forlenza OV, Laks J, Vale FAC. Tratamento da doença de Alzheimer: recomendações e sugestões do Departamento Científico de Neurologia Cognitiva do Envelhecimento da Academia Brasileira de Neurologia. Arq Neuropsiquiatr. 2005; 63(4):1104-12.

36. Letenneur L, Gilleron V, Commenges D, Helmer C, Orgogozo JM, Dartigues JF. Are sex and educational level independent predictors of dementia and Alzheimer's disease? Incidence data from the PAQUID Project. J Neurol Neurosurg Phsychiatry. 1999; 66(2):177-83.

37. Lee JY, Chang SM, Jang HS, Chang JS, Suh $\mathrm{GH}$, Jung $\mathrm{HY}$, et al. Illiteracy and the incidence of Alzheimer's disease in the Yonchon County survey, Korea. Int. Psychogeriatr. 2008; 20(5):976-85.

RECEBIDO EM: 25/08/2008

ACEITO EM: 06/10/2009

Endereço para correspondência:

Patrícia Gomes de Azevedo

Av. Mauá, 1372

Araraquara - SP

CEP: 14801-190

E-mail: patylok12@hotmail.com 